\title{
COARSENING AND SELF-ORGANIZATION IN DILUTE DIBLOCK COPOLYMER MELTS AND MIXTURES
}

\author{
KARL GLASNER RUSTUM CHOKSI
}

\begin{abstract}
This paper explores the evolution of a sharp interface model for phase separation of copolymers in the limit of low volume fraction. Particles both exchange material as in usual Ostwald ripening, and migrate because of an effectively repulsive nonlocal energetic term. Coarsening via mass diffusion only occurs while particle radii are small, and they eventually approach a finite equilibrium size. Migration, on the other hand, is responsible for producing self-organized patterns.

We construct approximations based upon an ansatz of spherical particles similar to the classical LSW theory to derive finite dimensional dynamics for particle positions and radii. For large systems, kinetic-type equations which describe the evolution of a probability density are constructed. For systems larger than the screening length, we obtain an analog of the homogenization result of Niethammer \& Otto (Calc. Var. and PDE, Vol. 13 (2001)). A separation of timescales between particle growth and migration allows for a variational characterization of spatially inhomogeneous quasi-equilibrium states.
\end{abstract}

Ostwald ripening, a coarsening process described by the exchange of material between particles of the minority phase in binary mixtures (cf. [30, 17]), has been the topic of extensive study over the last 20 years. Much of the analytical work is based upon the Cahn-Hilliard equation which was conceived to model phase separation in binary alloys (cf [3]). This equation describes spinodal decomposition of a fine grained mixture and nucleation of phases rich in each component of the mixture. This is followed by interface coarsening via the Mullins-Sekerka law, which produces coarsening behavior with a wellestablished rate of $t^{1 / 3}$, i.e. the length scale of increases as a power law with exponent $1 / 3$.

In the dilute regime, the minority phase forms particles which interact through mass diffusion, and the resulting process is termed Ostwald ripening. One can derive both finitedimensional dynamics of individual particles, and in the limit of large particle number kinetic-type statistical descriptions ([16, 31, 30, 17, 19] and references therein). These descriptions provide a counterpart to rigorous upper bounds (e.g. [15, 24]) which predict and confirm temporal scaling laws.

The Cahn-Hilliard approach applies equally well to phase separation of mixtures or melts of homogeneous polymers which are thermodynamically incompatible [9]. Block copolymers, on the other hand, are inhomogeneous chain molecules composed of two or more monomer types (see Figure 1 top). Below a critical temperature, weak local repulsion of different species induces phase separation. Complete separation does not occur, however, because of molecular attachment of distinct monomer chains. Instead, a remarkable array

Key words and phrases. diblock copolymers; Ostwald ripening; coarsening, self-organization. 

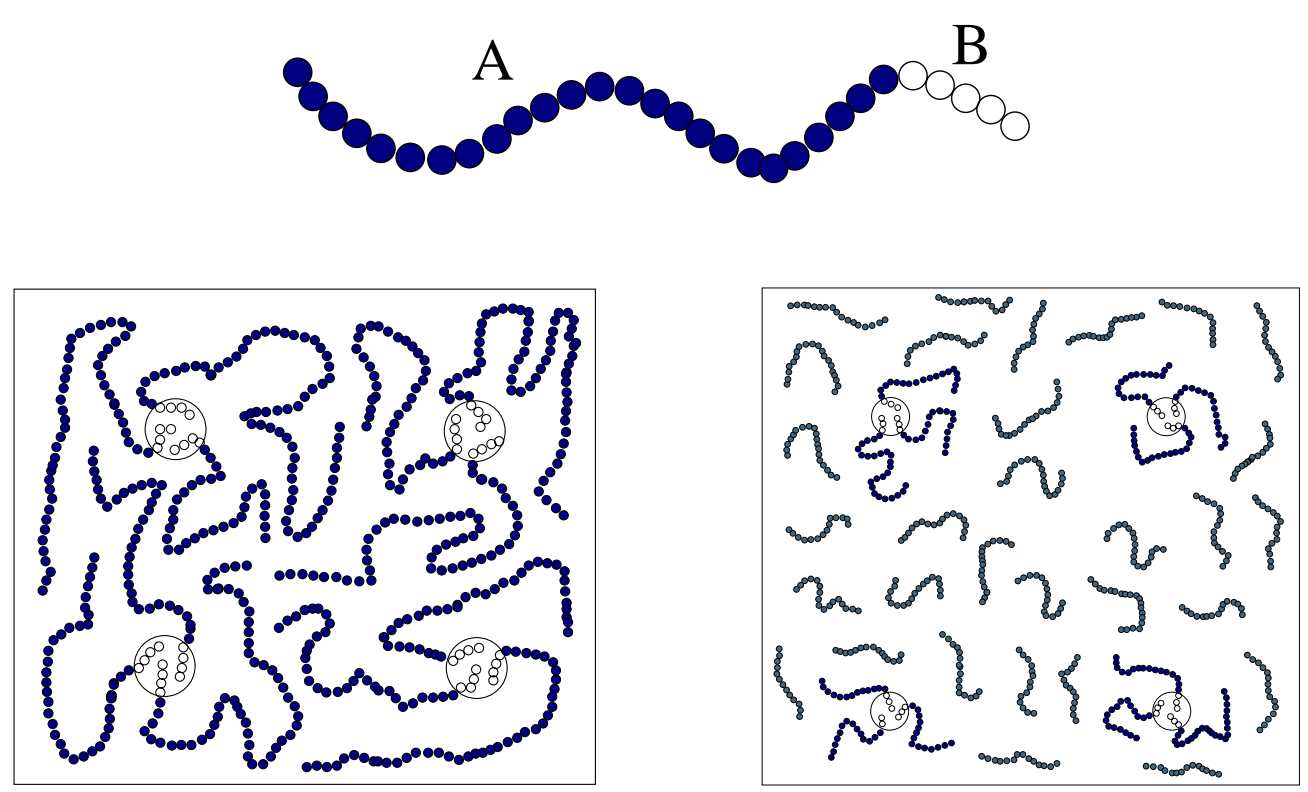

Figure 1: Top: an AB diblock copolymer macromolecule of small molecular weight. Bottom left: microphase separation of these diblock copolymers. Bottom right: phase separation in a mixture/blend of diblock copolymers and homopolymers of another monomer species having relatively weak interactions with the $\mathrm{A}$ and $\mathrm{B}$ monomers.

of locally periodic "metastable" states appear: lamellar, cylindrical, spherical, gyroid structures have all been observed $[1,12]$. Because of their variable architecture, these materials are extremely versatile, having applications in nanotechnology, lithography, photonics and controlled drug delivery [1].

This paper studies the modification to the usual coarsening process that results from long range interactions in systems of block copolymers. Our approach is via a modified Cahn-Hilliard equation which arises as a gradient flow (with respect to the $H^{-1}$ metric) of a nonlocal Cahn-Hilliard-type functional first introduced by Ohta and Kawasaki in [23]. We are interested in the strong segregation regime wherein $A / B$ interfaces are rather sharp in comparison to domain size, and hence focus on the sharp interface limit of this equation (cf. [21]) in the case of low volume fraction. That is, we consider diblock copolymers of very small molecular weight (ratio of $B$ monomers to $A$ ) and envisage either a melt of such diblock copolymers (cf. Figure 1, bottom left) or a mixture/blend of such diblocks with homopolymers of either type $A$ or of type $C$ but for which the $C A$ and $C B$ interactions are relatively weak (cf. Figure 1, bottom right).

This paper uses a plan of attack tangential to that for mixtures described by the ordinary Cahn-Hilliard equation. Sagui \& Desai [27] considered an abstract prototypical model for these systems in two dimensions and studied the reduced dynamics numerically. We follow a similar reduction scheme specialized to the copolymer case in three dimensions, and go ever further to characterize the evolution statistically. In particular, we base our analysis 
upon an ansatz of small spherical regions (inclusions) for the minority monomer phase and utilize the following program:

(i) We identify two spatial scales, the screening length and the structure length, and the temporal regimes relevant for the evolution process of small spherical domains. The screening length is, roughly speaking, the distance over which particles interact and hence its relation to the sample size is important. The structure length is the intrinsic scale set by the competition of short and long range interactions which comprise the free energy. The temporal scales correspond respectively to traditional Ostwald ripening (coarsening), structure formation when the size of the inclusions stabilizes, and finally particle migration or pattern formation. Based upon the size of screening length relative to the sample size, we focus on two separate scenarios: unscreened and screened systems.

(ii) For the unscreened case, we modify the classical LSW (for Lifshitz \& Slyozov [16] and Wagner [31]) theory to derive systems of effective ordinary differential equations for both the radii and the centers of the small spheres. These ODEs capture both the separation of time scales as well as the length scale corresponding to the structured regime. We characterize these equations variationally by connecting them to a reduced free energy.

(iii) For screened systems, we again apply the LSW arguments to derive ODEs for the particle radii and a kinetic equation for the particle radii distribution. We also perform a homogenization of the Poisson equation relating the potential to the spatial distribution on particles. This allows for an effective homogenized system which couples the kinetic equation with the homogenized Poisson equation, and extends a homogenization result of Niethammer \& Otto [19]. One can then study stability of steady states of this system under certain constraints via a homogenized energy.

(iv) To address the last stage of particle migration, we develop the basic approximations one step further, which allows for additional approximation of the free energy to include a particle interaction term. We explore the consequence of this subdominant term on particle migration and pattern formation.

Our results are consistent with a recent Gamma-convergence study [5] of free energy in the small volume fraction limit, and a recent study [25] of stable local minimizers for small volume fraction limit. The Gamma-convergence study is based on the small volume fraction asymptotics of the (suitably rescaled) free energy, and reveals at leading order, a local energy responsible for the first stages of particle evolution, i.e., the coarsening process. At the next order, one finds an interaction energy responsible for the last stage of particle migration, i.e., self-organization. We discuss the connections in Sections 3.4 and 5.2. 


\section{The Modified Cahn-Hilliard model and its sharp interface limit}

A density function theory $[23,7]$ for the relative monomer fraction $u: \Omega \rightarrow[0,1]$ yields a free energy of the form

$$
\begin{aligned}
F(u) & =\int_{\Omega} \frac{\epsilon^{2}}{2}|\nabla u|^{2}+W(u)+\frac{\sigma}{2}|\nabla v|^{2} d x \\
\Delta v & =u-\rho, \quad \rho=\frac{1}{|\Omega|} \int_{\Omega} u d x .
\end{aligned}
$$

where the second term is a double-well local energy preferring $u=0$ and $u=1$, and the last term describes nonlocal interactions while conserving volume fraction $\rho$. Dynamical models can be built using a mass-conserved gradient descent method. The most natural of which is gradient flow within the Hilbert space $H^{-1}$, and leads to the following modification of the well-known Cahn-Hilliard equation

$$
u_{t}=\Delta\left(-\epsilon^{2} \Delta u+W^{\prime}(u)\right)-\sigma(u-\rho) .
$$

The singular limit $\epsilon \rightarrow 0, \sigma \sim \epsilon$ is of particular interest [21, 10, 14], and as first noted by Nishiura and Ohnishi, it leads to a free boundary evolution which we will use as a point of departure.

Let the physical domain $\Omega$ be divided into two non-overlapping phase domains $\Omega^{+}, \Omega^{-}$ where $\Omega^{+}$will denote the minority phase. The free boundary, denoted $\partial \Omega^{+}$, evolves according to

$$
\begin{aligned}
\Delta v & =\gamma \begin{cases}1-\rho & \text { in } \Omega^{+} \\
-\rho & \text { in } \Omega^{-}\end{cases} \\
v & =\kappa \text { on } \partial \Omega^{+} \\
V_{n} & =[\partial v / \partial n]_{+}^{-}
\end{aligned}
$$

where the convention is used that the normal velocity $V_{n}$ and mean curvature $\kappa$ are outward with respect to $\Omega^{+}$. For simplicity, we take $\Omega=[0,1]^{3}$ and assume periodic boundary conditions throughout. The volume fraction of the dilute phase is $\left|\Omega^{+}\right|=\rho$; we will be concerned about the case $\rho \ll 1$.

The free energy corresponding to $(3-5)^{1}$ is

$$
F=\int_{\partial \Omega^{+}} d A+\frac{\gamma}{2} \int_{\Omega}|\nabla w|^{2} d x,
$$

where $w$ is a continuously differentiable solution (unique up to a constant) of

$$
\Delta w= \begin{cases}1-\rho & \text { in } \Omega^{+} \\ -\rho & \text { in } \Omega^{-}\end{cases}
$$

\footnotetext{
${ }^{1}$ Alternatively, the free energy is, modulo a constant, the $\Gamma$-limit of (1) as $\epsilon$ tends to 0 where $\sigma=\epsilon \gamma$.
} 
The free boundary problem has a gradient flow structure; in particular

$$
\frac{d F}{d t}=-\int_{\Omega}|\nabla \mu|^{2} d x
$$

where $\mu=v-\gamma w$; physically $\mu$ is the chemical potential that gives rise to the material flux $-\nabla \mu$.

\section{SCAling REgImes of THE LOW-VOLUme FRACTION LIMIT}

We suppose that the minority phase is described to a good approximation by a collection of non-overlapping spheres (hereafter "particles")

$$
\Omega^{+}=\cup_{i=1}^{N} B_{R_{i}}\left(x_{i}\right) .
$$

The reasonableness of this starting point hinges on two conditions: (1) spherical particles are, in some sense, stable equilibria, and (2) relaxation to these equilibria is faster than the dynamics of particle-particle interaction. Since the energy is nonlocal, it is impossible to regard an isolated particle as an equilibrium of the complete free boundary problem. However, in the low volume fraction limit, the energy of interaction between particles is subdominant to the particles "self-energy", and a simplified variational problem for equilibrium and stability can be considered (see Appendix A).

Let $R$ be the typical sphere radius and $d$ the minimum pairwise distance (see Figure 2 ). We assume throughout that $R \ll d$ which implies that we are in the small volume fraction regime:

$$
\rho \sim(R / d)^{3} \ll 1
$$

There are two other derived length scales which distinguish the regimes to be investigated.

2.1. Spatial scales. One important scale is the screening length $\xi$, which roughly sets the distance over which particles interact. From a mathematical point of view, screening arises in solving elliptic Dirichlet problems in domains which are highly "perforated". It is understood that Green's functions on these domains have an exponentially decreasing character [20], with $\xi$ setting the length scale of exponential decrease. Niethammer \& Otto [19] show that the screening length for the dilute particle scenario scales as

$$
\xi \sim \frac{d^{3 / 2}}{R^{1 / 2}} \sim(N R)^{-1 / 2}
$$

where $N$ is the number of particles per unit volume. In our problem, interactions are screened when the screening length is similar or less than the system size. We later show how this length scale arises naturally in our formal approximations.

A second scale is set by the competition between local and nonlocal effects encoded in the parameter $\gamma$. The (non-dimensional) length $\gamma^{-1 / 3}$ sets the scale for the radii of nearequilibrium particles. In particular, we show that particle sizes typically approach a radius $R_{*}=(3 / \gamma)^{1 / 3}$, which is smaller than the threshold $R_{s}$ for stability. The relative size of $R$ and $\gamma^{-1 / 3}$ therefore delineates two regimes that are characterized by the dominant balance of terms in (3). 


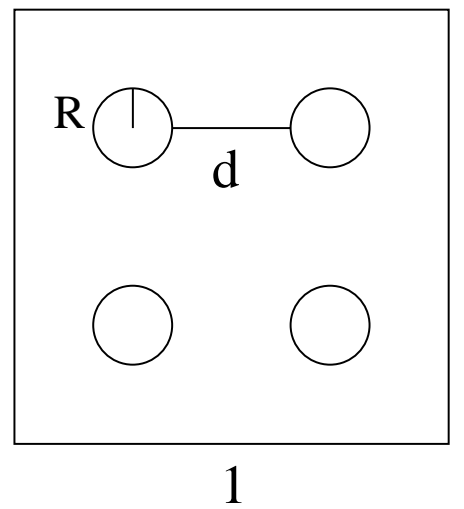

Figure 2: Collection of particles with fixed small volume fraction $(\sim R / d)$ where $R$ denotes the radius of the spheres and $d$, the separation distance, and sample size is set to unity.

Outside of the particle regions $\Omega_{+}$, the solution $v$ to (3) varies over scales comparable to $\xi$. This implies the scaling

$$
\Delta v \sim \frac{R^{-1}}{\xi^{2}} \sim d^{-3}
$$

If

$$
R \ll \gamma^{-1 / 3} \quad \text { (Coarsening regime) }
$$

dominant balance in $\Omega_{-}$gives $\Delta v=0$, and the situation is similar to the usual description of Ostwald ripening. The other case to consider is

$$
R \sim \gamma^{-1 / 3} \quad \text { (Structured regime) }
$$

where dominant balance in $\Omega_{-}$gives $\Delta v=-\gamma \rho$. This situation is predominantly characterized by equilibration of particle radii to a range of stable values, rather than coarsening.

2.2. Scaling in time. There are several temporal regimes, each characterized by a certain scaling:

- Coarsening: when $R \ll \gamma^{-1 / 3}$, the dynamics coincide with traditional Ostwald ripening, where the relevant timescale is $\tau \sim R^{3}$.

- Structure formation: when $R \sim \gamma^{-1 / 3}$, particle sizes equilibrate (at least locally). This equilibration also occurs on the timescale $\tau \sim R^{3}$. We will derive approximations on this timescale which describe relaxation of particle radii to locally constant values. Steady states of this evolution do not correspond to minimizers of the exact energy (6), but rather to low energy states which are energy minimizers of a restricted problem that ignores particle migration. In the case of small screening length, there can be a spatially inhomogeneous ("polydisperse") distribution of particle sizes. A similar interplay between spatial distribution and size was described in numerical experiments of Sagui \& Desai [27]. 
- Pattern formation: This stage of evolution is described by particle migration and organization of roughly periodic patterned states, principally resulting from repulsive nonlocal energy terms. The relevant timescale is $\tau \sim R d^{3}$.

\section{UNSCREENED SYSTEMS}

We first describe the approximate dynamics in the simplest case when the screening length is large compared with the system size. The particle number and inter-particle distance therefore have the scales

$$
N \sim d^{-3} \sim \xi^{-2} R^{-1} \ll R^{-1}
$$

The intermediate coarsening behavior can be captured by modifying the classical LSW theory (developed independently by Lifshitz \& Slyozov [16] and Wagner [31]).

3.1. The LSW argument and its modification. Provided the radii satisfy $R \ll \gamma^{-3}$, dominant balance in equation (3) means that in $\Omega^{-}, v$ satisfies the Laplace equation $\Delta v=0$ to leading order. The traditional LSW approximation assumes a spatially infinite domain; here we adjust the argument using the modified Green's function $G_{p}$ that formally satisfies

$$
-\Delta G_{p}(x)=\delta(x)-1 /|\Omega|
$$

subject to periodic boundary conditions (Appendix B provides a review of the Ewald method for practical computation of this Green's function). Note that $G_{p}$ behaves like the free space Green's function in the near field:

$$
G_{p}\left(x-x_{i}\right)=\frac{1}{4 \pi\left|x-x_{i}\right|}+O(1), \quad\left|x-x_{i}\right| \rightarrow 0 .
$$

The fundamental approximation takes the form

$$
v(x)=A_{0}+\sum_{i=1}^{N} A_{i} G_{p}\left(x-x_{i}\right)+O(1), \quad R \rightarrow 0 .
$$

where the coefficients $A_{0}, A_{i}$ are assumed to be $O\left(R^{-1}\right), O(1)$ respectively for small $R$. In light of (10), there is a solvability constraint on the coefficients

$$
\sum_{i=1}^{N} A_{i}=0
$$

which guarantees $\Delta v=0$. The validity of the ansatz (12) in the context of Ostwald ripening has been the subject of recent work (cf. [17, 19]). In general, one should regard this as an "outer" or mean-field solution valid away from the free boundaries. At leading order, however, (12) also satisfactorily describes the boundary layer near each particle. The case of particle migration (Section 5), on the other hand, requires a correction term to the boundary layer. 
Applying the boundary condition on $v$ and using (11) gives asymptotically for small $R$

$$
\frac{2}{R_{i}}=A_{0}+\frac{A_{i}}{4 \pi R_{i}}+\sum_{j \neq i}^{N} A_{j} G_{p}\left(x_{i}-x_{j}\right)+O(1) .
$$

We can estimate the size of the summation by observing that for roughly homogeneous distributions of particles, it behaves like an integral

$$
\sum_{i \neq j}^{N} A_{j} G_{p}\left(x_{i}-x_{j}\right) \approx \frac{1}{d^{3}} \int_{\Omega} A(x) G_{p}\left(x_{i}-x\right) d x=O\left(d^{-3}\right)=O\left(R^{-1}\right)
$$

where $A\left(x_{i}\right) \approx A_{i}$. By virtue of (9), the dominant part of (14) is

$$
\frac{2}{R_{i}}=A_{0}+\frac{A_{i}}{4 \pi R_{i}} .
$$

For each particle in the minority phase $\Omega^{+}, \Delta v \approx \gamma$, and the solution has the form

$$
v(x)=C_{0}+\gamma\left|x-x_{i}\right|^{2} / 6, \quad x \in B_{R_{i}}\left(x_{i}\right) .
$$

In the context of the approximation, the free boundary velocity given by (5) is radially symmetric at leading order, and gives the dynamics of the particle radii as

$$
\frac{d R_{i}}{d t}=[\partial v / \partial n]_{+}^{-} \approx-\frac{A_{i}}{4 \pi R_{i}^{2}}-\frac{\gamma R_{j}}{3}
$$

For $R \ll \gamma^{-3}$, the second term in (18) is also small, leading to

$$
\frac{d R_{i}}{d t}=\frac{R_{i} A_{0}-2}{R_{i}^{2}} .
$$

Using (13), (16) and summing over $i$ leads to

$$
A_{0}=\frac{2 N}{\sum_{i=1}^{N} R_{i}} .
$$

Equations (19-20) are the traditional LSW approximation, which only holds in our system for large screening lengths and radii much smaller less than the structure length scale.

When $R \sim \gamma^{-3}$, dominant balance yields the Poisson equation $\Delta v=-\gamma \rho$ in the exterior region $\Omega^{-}$. The monopole ansatz (12) can still be used, but the solvability condition on the coefficients changes:

$$
v(x) \approx A_{0}+\sum_{i=1}^{N} A_{i} G_{p}\left(x-x_{i}\right), \quad \sum_{i=1}^{N} A_{i}=-\gamma \rho .
$$

Using (16) and the fact that $\gamma \sim R^{-1 / 3}$, it follows that to leading order

$$
\frac{d R_{j}}{d t}=\frac{R_{j} A_{0}-2-\gamma R_{j}^{3} / 3}{R_{j}^{2}}
$$


Summing over all $i$ in (16) and using the solvability constraint in (21) leads to

$$
A_{0}=\frac{2 N}{\sum_{i=1}^{N} R_{i}}+\frac{\gamma}{3} \frac{\sum_{i=1}^{N} R_{i}^{3}}{\sum_{i=1}^{N} R_{i}} .
$$

Equations (22-23) are a modification of the traditional LSW approximation in the structured regime. The remainder of this section will discuss their behavior.

3.2. Behavior of the modified LSW evolution. The system $(22,23)$ reduces to the classical LSW approximation for $\gamma=0$. In that case, $A_{0} / 2$ is the reciprocal of the average particle radius, and the interpretation of (22) is simple: particles larger than the average will grow, while the smaller ones will shrink.

The situation for $\gamma>0$ is more involved. From the form of (22) it is clear that particles which are very small or large relative to the average will shrink, whereas particles with radii in the "growth" interval

$$
I_{g}\left(A_{0}\right)=\left[R_{-}\left(A_{0}\right), R_{+}\left(A_{0}\right)\right], \quad R_{ \pm} \text {are positive roots of } R_{j} A_{0}-2-\gamma R_{j}^{3} / 3 .
$$

have $d R / d t \geq 0$. Note that this interval cannot be empty, otherwise all radii would shrink and volume would not be conserved.

3.2.1. Small particle initial conditions. Suppose that initially all particle radii are very small, so that the first term in the expression for $A_{0}$ dominates. As coarsening proceeds, $A_{0}$ will diminish along with the size of the interval $I_{g}$. Ultimately, most particles will have either vanished or have radii close to this shrinking interval, which is itself vanishing as a critical value $A_{0}^{*}$ of $A_{0}$ is approached. Near this point, most particles must have similar radii which will converge to a common value $R_{*}$ when the interval $I_{g}$ just vanishes. This happens when the growth rate $R_{*} A_{0}-2-\gamma R_{*}^{3} / 3$ undergoes a saddle-node bifurcation (relative to the parameter $A_{0}$ ), given by

$$
R_{*} A_{0}^{*}-2-\gamma R_{*}^{3} / 3=0, \quad \frac{d\left(R_{*} A_{0}^{*}-2-\gamma R_{*}^{3} / 3\right)}{d R_{*}}=0,
$$

which has the solution $A_{0}^{*}=\gamma R_{*}^{2}$ where the critical radius is

$$
R_{*}=\left(\frac{3}{\gamma}\right)^{1 / 3} .
$$

Of course, a small fraction of particles may have radii larger than the growth interval. These particles will lose mass as as particles in $I_{g}$ grow. If these two sets of particles reach equilibrium before $I_{g}$ vanishes, then the equilibrium radius may be somewhat larger than $R_{*}$. This possibility is investigated more deeply by considering energetic stability (section $3.3)$.

To illustrate the behavior for small particle initial conditions, we have simulated $(22,23)$ with particles sizes chosen from a uniform random distribution in the interval $R \in\left[0, R_{*} / 10\right]$. The reported behavior is more or less the same for any randomization. After a transient period, the particle radii distribution is peaked, and the average particle radius increases 

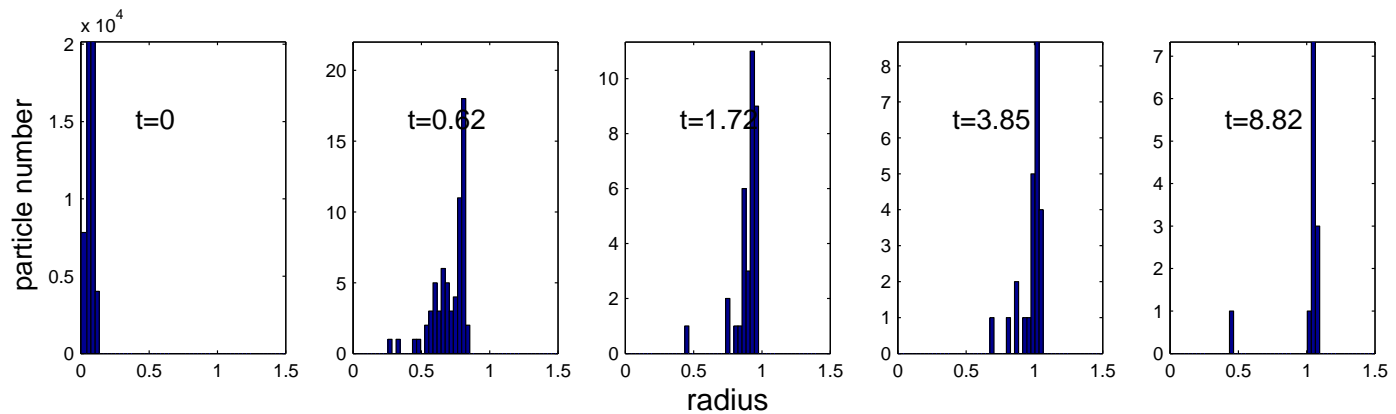

Figure 3: Evolution of the reduced dynamics of equations (22),(23). There were initially $10^{6}$ particles with radii chosen from a uniform random distribution of $R \in\left[0, R_{*} / 10\right]$. The critical radius was chosen to be $R_{*}=1$. Toward the end of the simulation (rightmost frame) most particles have radii near $R_{*}$. Eventually all radii converge to the same value (not shown).

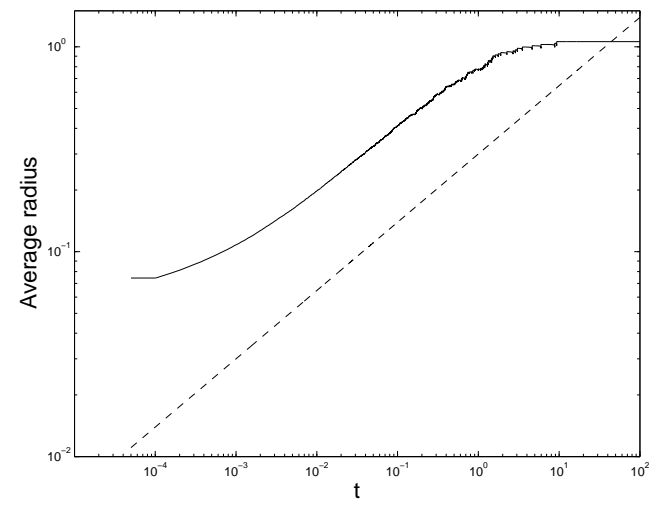

Figure 4: Average radius for the evolution $(22),(23)$ with small particle initial conditions. The dashed line with slope $1 / 3$ is provided for comparison. Initially, the usual coarsening scaling $R \sim t^{1 / 3}$ is observed, but slows when radii are comparable to $R_{*}$.

until it is just slightly larger that $R_{*}$ (Figure 3 ). We also compute the average radius (Figure 4). Initially, the coarsening process follows the expected LSW scaling, but saturates when particle sizes are on the same order as the structural length.

3.2.2. Arbitrary initial conditions. If radii are not all small initially, there may be a substantial fraction above the growth interval $I_{g}$. Ultimately most radii should converge to a common value $\bar{R} \approx R_{+}\left(A_{0}\right)$ since particles just bigger than $R_{+}\left(A_{0}\right)$ will shrink, while those just smaller will grow. This is possible for any value of $\bar{R}$ which is the larger of the two 

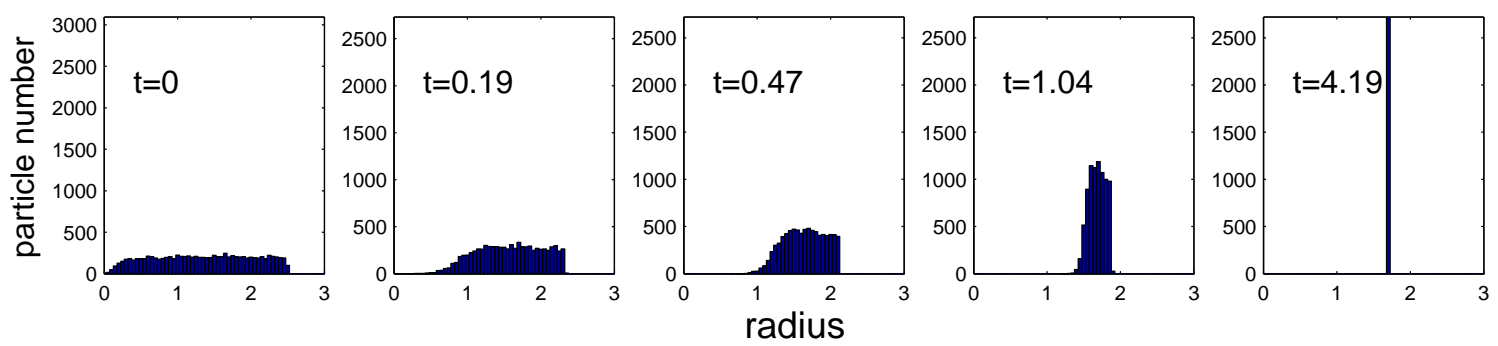

Figure 5: Evolution of (22),(23) with radii chosen from a uniform random distribution of $R \in\left[0,2.5 * R_{*}\right]$. The asymptotic behavior is toward an equilibrium radius somewhat larger than $R_{*}=1$.

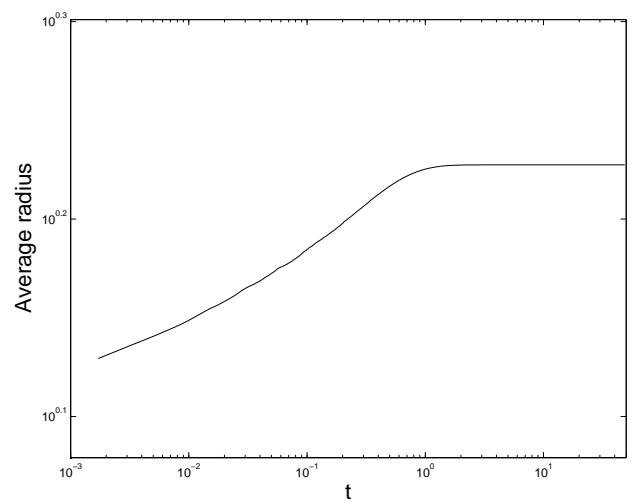

Figure 6: Average radius for the evolution (22),(23) with large random initial conditions.

roots of $f(R)=R A_{0}-2-\gamma R^{3} / 3$, which can be shown to satisfy

$$
\bar{R} \geq\left(\frac{3}{\gamma}\right)^{1 / 3}=R_{*} .
$$

There is therefore a range of stable long term behavior depending on initial conditions, characterized by convergence of particle radii to a common radius $\bar{R} \geq R_{*}$.

Simulations of $(22,23)$ for arbitrary initial conditions show similar coarsening of small particles (Figure 5), but the average radius converges to a value somewhat larger than $R_{*}$ (Figure 6).

3.3. Energetic reasoning. The behavior described above can also be explained in terms of an approximation of the system energy. In particular, we can show that within the context of the LSW approximation, an assembly of particles of common radius $R$ is energetically stable only if and only if $R \geq R_{*}$.

The self-energy of an isolated, spherical particle of radius $R$ can be regarded as the sum of the surface energy $4 \pi R^{2}$ and the nonlocal contribution in (6). The solution to (7) for 
$\rho \approx 0$ is approximated in a way similar to $v$. The difference is that instead of a Dirichlet boundary condition, there is Neumann boundary condition that requires continuity of the normal derivative. This gives the solution (up to additive constants)

$$
w= \begin{cases}|x|^{2} / 6-R^{2} / 2 & |x|<R \\ -\frac{R^{3}}{3|x|} & |x|>R\end{cases}
$$

One then computes the total self-energy of a particle as

$$
4 \pi R^{2}+\frac{\gamma}{2} \int_{\mathbb{R}^{3}}|\nabla w|^{2} d x=4 \pi\left(\frac{\gamma R^{5}}{15}+R^{2}\right) .
$$

The system $(22,23)$ is in fact a gradient flow. The self-energy of the collection of particles is

$$
E_{s}=\sum_{i=1}^{N} 4 \pi\left(\frac{\gamma R_{i}^{5}}{15}+R_{i}^{2}\right)
$$

so that

$$
\begin{aligned}
\frac{d E_{s}}{d t} & =4 \pi \sum_{i=1}^{N}\left(\frac{\gamma R_{i}^{4}}{3}+2 R_{i}\right) \frac{d R_{i}}{d t} \\
& =-4 \pi \sum_{i=1}^{N} R_{i}^{3}\left(\frac{d R_{i}}{d t}\right)^{2},
\end{aligned}
$$

where (22) and conservation of mass $\sum_{i} R_{i}^{2} d R_{i} / d t=0$ was used. Using the Riemannian metric given by the bilinear form

$$
\left(\varphi_{1}, \varphi_{2}\right)_{g} \equiv 4 \pi \sum_{i=1}^{N} R_{i}^{3} \varphi_{1} \cdot \varphi_{2}, \quad \varphi_{1,2} \in \mathbb{R}^{n}
$$

then $(22,23)$ satisfy the definition for a generalized gradient flow $\nabla E_{s} \cdot \varphi=(d R / d t, \varphi)_{g}$ for all (volume conserving) $\varphi \in \mathbb{R}^{n}$, where $R=\left(R_{1}, R_{2}, \ldots, R_{N}\right)$.

The metric corresponds to the dissipation of energy (8) in the limit of small volume fraction. Indeed, suppose $-\nabla \mu$ is the flux of material resulting from a prescribed, mass conserving radial dynamics $d R_{i} / d t$. Then $\mu$ solves a free boundary problem

$$
\Delta \mu=0, \quad[\partial \mu / \partial n]_{+}^{-}=-\frac{d R_{i}}{d t} \text { on each } \partial B_{i}
$$

Then $\mu \approx C_{i} /\left|x-X_{i}\right|+C_{2}$ outside of each particle so that $C_{i}=-R_{i}^{2}\left(d R_{i} / d t\right)$. Inside, $\mu \approx 0$, therefore in the small volume fraction limit the dissipation is

$$
\int_{\Omega}|\nabla \mu|^{2} d x=\sum_{i=1}^{N} \int_{\left|x-X_{i}\right|>R_{i}} \frac{C_{i}^{2}}{\left|x-X_{i}\right|^{4}} d x=4 \pi \sum_{i=1}^{N} R_{i}^{3}\left(\frac{d R_{i}}{d t}\right)^{2},
$$

which agrees with (29). 
It is easiest to compute the energetic stability of a collection of spherical particles $\left\{B_{R_{i}}\left(x_{i}\right)\right\}$ by working with the volume variables $V_{i}=4 \pi R_{i}^{3} / 3$. The energy of this collection is, according to our above approximations, given by

$$
E_{s} \propto \sum_{i=1}^{N}\left[\frac{\gamma V_{i}^{5 / 3}}{15}+V_{i}^{2 / 3}\right] .
$$

Critical points of the energy are subject to the volume conservation constraint

$$
\sum_{i=1}^{N} V_{i}=\text { constant. }
$$

Therefore the only critical points are where particles have the same size, i.e. $V_{i}=V=$ a constant. The second variation of the energy (the Hessian of $E$ ) must be positive definite on the affine linear subspace defined by (34) for the particles to energetically stable. In this case the Hessian is just a diagonal matrix with diagonal entries proportional to $V^{-1 / 3} / 27-$ $V^{-4 / 3} / 9$. The critical point of $E_{s}$ is therefore a energy minimum only if this quantity is positive, which means $V>3 / \gamma$, or in terms of the particle radii,

$$
R>\left(\frac{3}{\gamma}\right)^{1 / 3}=R_{*}
$$

which agrees with the earlier discussion.

\subsection{Connection with the Leading Order Gamma-convergence of the Energy.} We show here how the self-energy (28), used in the previous subsection, is consistent with a recent asymptotic study [5, 6] of the full free energy (6) in the limit where the volume fraction $\rho$ tends to zero, and $\gamma$ is suitably scaled so that the eventual number of particles remains positive. In this study, one finds at leading order, a local self-energy analogous to (28) which drives the limited-time coarsening process. At the next level, one finds a Coulomb-like interaction between particles, similar to (75), which is responsible for the particle migration and pattern formation addressed in Section 5. There is a natural language in which to present the rigorous asymptotic analysis, that of Gamma-convergence [2] (often written as $\Gamma$-convergence). The rigorous Gamma-convergence results in 2D and $3 \mathrm{D}$ are presented in [5]. Here, let us give a few details on the formal decomposition of the (3D) free energy and the resulting asymptotic limiting contributions. See [5] for precise treatment with explicit representation of the Gamma-limits.

By addition of a constant, we can assume that the modified Green's function $G_{p}$ satisfies

$$
\int_{\Omega} G_{p}(x-y) d y=0, \quad \forall x \in \Omega
$$

Hence we may rewrite the energy in (6) as:

$$
E(u):=\int_{\Omega}|\nabla u|+\frac{\gamma}{2} \int_{\Omega} \int_{\Omega} G_{p}(x-y) u(x) u(y) d x d y
$$


where $u \in B V(\Omega,\{0,1\})$, a characteristic function of bounded variation. Thus the first term is simply the area of the interface $\partial \Omega^{+}$. We are interested in minimizing this energy over all such $u$ with average $\rho$, and deriving an effective energy in the limit wherein $\rho$ tends to 0 but the number of particles remains $O(1)$. The effective energy will be defined over weighted single point particles (Dirac delta masses). To this end, we must rescale and renormalized the energy. We introduce a new parameter $\eta$ controlling the (decreasing) volume fraction, i.e., $\rho=\eta M$ for some fixed $M$ reflecting the total mass of the limiting (point) particles. In order to enforce (cf. $[6,5])$ that the number of particles remains $O(1)$ as $\eta \rightarrow 0$, we slave $\gamma$ to $\eta$ via

$$
\gamma \sim 1 / \eta
$$

Setting

$$
v=u / \eta
$$

the energy can then be written as $E=\eta F_{\eta}$ where $F_{\eta}$ is defined for all $v \in B V(\Omega,\{0,1 / \eta\})$ by

$$
F_{\eta}(v):=\int_{\Omega}|\nabla v|+\frac{\gamma}{2} \int_{\Omega} \int_{\Omega} G_{p}(x-y) v(x) v(y) d x d y
$$

One readily checks (cf. [6]) that the minimum of $F_{\eta}$ scales like $\eta^{-1 / 3}$, and hence let us consider the asymptotics of $\eta^{1 / 3} F_{\eta}$.

The asymptotics are best illustrated by studying a minimizing sequence $v_{\eta}$ with the following form. Let $v_{\eta}=\sum_{i=1}^{N_{\eta}} v_{\eta}^{i}$, where each $v_{\eta}^{i}$ is of the form $v_{\eta}^{i}=\eta^{-1} \chi_{E_{\eta}^{i}}$, and the sets (called particles) $E_{\eta}^{i}$ are connected, non-intersecting with smooth boundary. We define the mass of each connected component as

$$
\alpha_{\eta}^{i}=\int_{\Omega} v_{\eta}^{i}=\frac{1}{\eta}\left|E_{\eta}^{i}\right|
$$

As $\eta \rightarrow 0$ we assume the following convergence (weak-* convergence in the sense of measures)

$$
v_{\eta} \longrightarrow \sum_{i} \alpha^{i} \delta_{x^{i}}, \text { where } \alpha_{\eta}^{i} \rightarrow \alpha^{i} \text { and } x^{i} \in \Omega
$$

Note that as $\eta \rightarrow 0$, the relative masses of the particles need not be uniformly distributed. 
The energy $F_{\eta}\left(v_{\eta}\right)$ of (36) then splits into two pieces:

$$
\begin{aligned}
\eta^{1 / 3} F_{\eta}\left(v_{\eta}\right)= & \eta^{1 / 3} \sum_{i}\left\{c_{0} \int_{\Omega}\left|\nabla v_{\eta}^{i}\right|+\int_{\Omega} \int_{\Omega} G_{p}(x-y) v_{\eta}^{i}(x) v_{\eta}^{i}(y) d x d y\right\} \\
& +\eta^{1 / 3} \sum_{(i, j): i \neq j} \int_{\Omega} \int_{\Omega} G_{p}(x-y) v_{\eta}^{i}(x) v_{\eta}^{j}(y) d x d y \\
= & \eta^{1 / 3} \sum_{i} F_{\eta}\left(v_{\eta}^{i}\right) \\
& +\eta^{1 / 3} \sum_{(i, j): i \neq j} \int_{\Omega} \int_{\Omega} G_{p}(x-y) v_{\eta}^{i}(x) v_{\eta}^{j}(y) d x d y .
\end{aligned}
$$

As $\eta$ tends to zero, the second term vanishes, since by (37)

$$
\sum_{(i, j): i \neq j} \int_{\Omega} \int_{\Omega} G_{p}(x-y) v_{\eta}^{i}(x) v_{\eta}^{j}(y) d x d y \longrightarrow \sum_{(i, j): i \neq j} \alpha^{i} \alpha^{j} G_{p}\left(x^{i}-x^{j}\right) .
$$

The first term is $O(1)$ as $\eta \rightarrow 0$, and is essentially the sum of the energies of each particle, i.e. there is no interaction between the particles and the leading order behavior is entirely local. In the language of Gamma-convergence, we have

$$
\eta^{1 / 3} F_{\eta}\left(v_{\eta}\right) \stackrel{\Gamma}{\longrightarrow} F_{0},
$$

where $F_{0}$ is defined over weighted Dirac masses $\sum_{i} \alpha^{i} \delta_{x^{i}}$, and consists of a sum of the individual energies for each particle of mass $\alpha^{i}$. These individual energies are defined variationally over characteristic functions of sets with volume $\alpha^{i}$, and involve perimeter plus the long-range self-interaction, the latter computed over the entire space $\mathbb{R}^{3}$.

In order to make a connection with our previous calculations, precisely with (28), let us consider $\eta$ small but positive and take the particles $E_{i}$ to be spheres of radii $R_{i}$ and overall volume fraction $\rho$. For $\eta$ small, the above implies that up to leading order, the renormalized energy associated with the collection of particles of radii $R_{i}$ can be replaced by $F_{0}$, which is the sum of the perimeter and self-interaction (defined over all space) of each spherical particle of approximate size $\alpha^{i}$. A simply scaling argument implies that

$$
F_{0} \sim \sum_{i} c_{1}\left(\alpha^{i}\right)^{2 / 3}+c_{2} \gamma\left(\alpha^{i}\right)^{5 / 3}
$$

for constants $c_{1}$ and $c_{2}$. Thus, at leading order,

$$
\begin{aligned}
E & =\eta F_{\eta} \\
& =\eta^{2 / 3}\left(\eta^{1 / 3} F_{\eta}\right) \\
& \sim \eta^{2 / 3} F_{0} \\
& \sim \eta^{2 / 3} \sum_{i}\left(\alpha^{i}\right)^{5 / 3}+\left(\alpha^{i}\right)^{2 / 3} .
\end{aligned}
$$


On the other hand, since

$$
R_{i} \sim\left(\eta \alpha^{i}\right)^{1 / 3}
$$

the self-energy of (28) can be written as

$$
\begin{aligned}
E_{s} & =\sum_{i} \gamma\left(\eta \alpha^{i}\right)^{5 / 3}+\left(\eta \alpha^{i}\right)^{2 / 3} \\
& =\sum_{i} \eta^{-1}\left(\eta \alpha^{i}\right)^{5 / 3}+\left(\eta \alpha^{i}\right)^{2 / 3} \\
& =\eta^{2 / 3} \sum_{i}\left(\alpha^{i}\right)^{5 / 3}+\left(\alpha^{i}\right)^{2 / 3}
\end{aligned}
$$

Thus the self-energy (28) is the leading order term of the full energy (6) in the regime of small volume fraction.

\section{ScReEning And homogenization}

If the screening length $\xi$ is similar or smaller than the system's size (set to unity here) only particles within a distance set by $\xi$ have significant interaction. This is in contrast to the unscreened case, where particle dynamics are determined entirely by a constant mean field (represented by the fact $v \approx A_{0}$ away from particles), which in turn depends on all particles in the system.

4.1. LSW approximation for large systems. We now look for an approximation under the scalings

$$
N \sim d^{-3} \gg R^{-1}, \quad R \sim \gamma^{-3}
$$

corresponding to a system size larger than the screening length. These approximations are the most general in the sense they reduce to the previous cases for small radii or large screening length.

The solution to $\Delta v=-\gamma \rho$ in the exterior region is given as in the unscreened case by (21). The principal difference is that the summation term in equation (14) is no longer subdominant. The same approximation of the boundary condition as before gives a linear system for the coefficients $A_{i}$ that reads

$$
\frac{2}{R_{i}}=A_{0}+\frac{A_{i}}{4 \pi R_{i}}+\sum_{j \neq i}^{N} A_{j} G\left(x_{i}-x_{j}\right)
$$

which is closed by the solvability condition

$$
\sum_{i=1}^{N} A_{i}=-\gamma \rho .
$$


The solution to $\Delta v \approx \gamma$ in the minority phase $\Omega^{+}$is the same as in (17). The dynamics arise in same fashion as the unscreened case (22), giving

$$
\frac{d R_{j}}{d t}=\frac{-A_{i} /(4 \pi)-\gamma R_{i}^{3} / 3}{R_{i}^{2}}
$$

4.2. Homogenization and kinetic equations. In the large number limit, it is useful to work with the (unnormalized) joint distribution $\nu(x, R)$ of particle positions and radii

$$
\int_{\Omega^{\prime}} \int_{R_{1}}^{R_{2}} \nu(x, R) d R d x=\#\left\{i \mid x_{i} \in \Omega^{\prime}, \quad R \in\left(R_{1}, R_{2}\right)\right\} .
$$

The goal is to derive an equation for $\nu$ that captures the evolution in a statistical fashion. It is convenient to define two related quantities, the number density and "electrostatic" capacity:

$$
\begin{aligned}
\phi(x) & \equiv \int \nu(x, R) d R=\sum_{i=1}^{N} \delta\left(x-x_{i}\right) \\
\mu(x) & \equiv \int R \nu(x, R) d R=\sum_{i=1}^{N} R_{i} \delta\left(x-x_{i}\right) .
\end{aligned}
$$

In the limit of a large number of small particles, the Dirichlet problem for $v$ can be homogenized to yield an effective equation for the limiting solution we denote by $\bar{v}(x)$ (cf. $[19,11])$. To see how this arises, note that for small radius, each particle has a boundary layer of thickness $R$, outside of which $v$ is well approximated by the mean field limit $\bar{v}(x)$. The boundary layer is described (at leading order) by a solution to Laplace's equation exterior to the $i$-th particle, which has the form

$$
v \sim v_{i}+\frac{A_{i}}{4 \pi\left|x-x_{i}\right|}
$$

Applying the boundary condition for $v$ gives the relationship

$$
v_{i}+A_{i} /\left(4 \pi R_{i}\right)=2 / R_{i}
$$

therefore the mean field near the $i$-th particle is given by

$$
\bar{v}\left(x_{i}\right) \approx v_{i}=\frac{2-A_{i} /(4 \pi)}{R_{i}} .
$$

The linear system (40) can therefore be written as

$$
A_{0}+4 \pi \sum_{j \neq i}^{N}\left(2-v_{j} R_{j}\right) G_{p}\left(x_{i}-x_{j}\right)=v_{i}
$$


By virtue of (44-45) and the approximation (47), the summations can be replaced by integrals:

$$
\begin{aligned}
\sum_{j \neq i}^{N} G_{p}\left(x_{i}-x_{j}\right) & =\int_{\Omega} \phi(y) G_{p}\left(x_{i}-y\right) d y \\
\sum_{j \neq i}^{N} v_{i} R_{i} G_{p}\left(x_{i}-x_{j}\right) & \approx \int_{\Omega} \bar{v}(y) \mu(y) G_{p}\left(x_{i}-y\right) d y
\end{aligned}
$$

The condition (41) can be written using (47) as

$$
4 \pi \sum_{i=1}^{N}\left(2-v_{i} R_{i}\right)=-\gamma \rho
$$

or similarly using (44-45),

$$
4 \pi \int_{\Omega}(2 \phi-\mu \bar{v}) d x=-\gamma \rho
$$

Therefore (48) becomes an integral equation

$$
A_{0}+4 \pi \int_{\Omega}(2 \phi(y)-\mu(y) \bar{v}(y)) G_{p}(x-y) d y=\bar{v}(x) .
$$

Although $\phi, \mu$ are defined as distributions in (44),(45), one imagines that in the large number limit $\phi / N$ and $\mu / N$ approximate sufficiently smooth bounded functions. Roughly speaking, this requires that particles are not "clumped" and are sufficiently distributed throughout the spatial domain. This seems likely in our problem, since particles repel one another (see section 5). In what follows, $\phi, \mu$ will be regarded as those smooth functions obtained in the large number limit.

Using the definition (10) of the modified Green's function, equation (53) is nothing more than an integral representation of the homogenized differential equation

$$
-\Delta \bar{v}+4 \pi \mu \bar{v}=8 \pi \phi+\gamma \rho .
$$

This equation is an extension of the homogenization result of Niethammer \& Otto [21].

In terms of the mean field, the dynamical approximation (42) can be written as

$$
\frac{d R_{i}}{d t}=\frac{\bar{v}\left(x_{i}\right) R_{i}-2-\gamma R_{i}^{3} / 3}{R_{i}^{2}} .
$$

Since $\bar{v}$ is determined entirely in terms of particle statistics by (54), the kinetic equation for $\nu$

$$
\nu_{t}+\partial_{R}\left(R^{-2}\left[R \bar{v}-2-\gamma R^{3} / 3\right] \nu\right)=0
$$

together with (54) forms a closed system for evolution of $\nu$. 
4.3. Stationary polydisperse states and energetics. For a specified (continuous) number distribution $\phi$, stationary states of the homogenized system $(54,56)$ can be sought. These states are not particle distributions which correspond to minimizers of the original energy (6), but rather should be thought of as constituting a lower-dimension manifold in state space on which the migration dynamics (see next section) are slow relative to the rate at which the manifold is approached. We can nevertheless characterize these states as energy minimizers by fixing $\phi$, which is equivalent to ignoring particle migration.

Since the mean field $\bar{v}$ is continuous, it follows that when (56) is stationary, nearby particles necessarily have the same radius; that is, we look for a distribution function $\nu$ which takes the form

$$
\nu(x, R)=\delta(R-\bar{R}(x)) \phi(x),
$$

where $\bar{R}(x)$ is the radii of particles at $x$. It follows that stationary solutions solve

$$
-\Delta \bar{v}=4 \pi \phi(2-\bar{R} \bar{v})+\gamma \rho, \quad \bar{v} \bar{R}-2-\frac{\gamma}{3} \bar{R}^{3}=0 .
$$

It is useful to introduce the quantity

$$
\bar{\rho}(x)=\frac{4 \pi}{3} \phi(x) \bar{R}(x)^{3},
$$

which represents the "local" volume fraction, so that (58(a)) can be written

$$
-\Delta \bar{v}=\gamma(\rho-\bar{\rho})
$$

Note that $\bar{R}$ and $\bar{\rho}$ can be used interchangeably.

The system (58) is best understood as the Euler-Lagrange equation for an energy which is the natural homogenized version of the system energy for prescribed number density $\phi$, namely

$$
E_{\text {hom }}(\bar{\rho} ; \phi)=4 \pi \int \phi\left(\frac{\gamma}{15} \bar{R}^{5}+\bar{R}^{2}\right) d x+\frac{\gamma}{2} \int_{\Omega}|\nabla w|^{2} d x, \quad-\Delta w=\rho-\bar{\rho} .
$$

The first term represents the self-energy (see equation (27)) of a collection of particles, whereas the second term is the nonlocal contribution deriving from variations in the mean field. The homogenized energy (60) is minimized for constant volume fraction, given by the constraint

$$
\int \bar{\rho} d x=\rho
$$

Note that one cannot rule out, a-priori, the possibility of $\bar{R} \leq 0$ for critical points of (60). This is a reflection of the fact that if the number density is locally too high, the coarsening dynamics will eliminate some particles so as to reduce the particle density before equilibrium is established. 
4.4. Stability of particle distributions in the homogenized limit. Recall that for the unscreened case, a collection of particles was stable (in the sense of the approximate energy) if all radii are larger than the critical radius $R_{*}$ (see equation $(35)$ ), which is a root of

$$
\eta\left(R_{*}\right)=0, \quad \eta(R) \equiv \frac{2 \gamma}{27 R}-\frac{2}{9 R^{4}}
$$

The homogenized energy turns out to have the same criteria:

$$
\bar{R}(x) \text { is a minimizer of } E_{\text {hom }} \quad \text { iff } \quad \bar{R}(x) \geq R_{*}, \quad x \in \Omega .
$$

The second variation of (60) is easily computed: it is just the second variation of the energy in the unscreened system plus a nonlocal term

$$
\begin{aligned}
& S\left(\rho^{\prime}\right)=\left.\frac{d^{2}}{d^{2} \epsilon}\right|_{\epsilon=0} E\left(\bar{\rho}+\epsilon \rho^{\prime}\right)= \\
& 4 \pi \int(4 \pi \phi / 3)^{-2} \eta(\bar{R})\left(\rho^{\prime}\right)^{2} d x+\frac{\gamma}{2} \int\left|\nabla w^{\prime}\right|^{2} d x, \quad \Delta w^{\prime}=\rho^{\prime} .
\end{aligned}
$$

where $\rho^{\prime}$ must be volume preserving so $\int \rho^{\prime} d x=0$.

To see why (61) is true, first note that the nonlocal term in the second variation (62) is always positive and therefore stabilizing. Therefore $\bar{R}(x) \geq R_{*}$ implies $\eta(\bar{R})>0$ and the entire second variation is positive. Conversely, suppose that $\bar{R}\left(x_{0}\right)<R_{*}$ for some $x_{0}$ so that $\eta\left(\bar{R}\left(x_{0}\right)\right)<0$, and consider for small $\epsilon>0$ the volume preserving perturbation

$$
\rho^{\prime}= \begin{cases}\frac{1}{\epsilon^{3}} & \left|x-x_{0}\right| \leq \epsilon \\ -\frac{1}{7 \epsilon^{3}} & \epsilon<\left|x-x_{0}\right|<2 \epsilon \\ 0 & \left|x-x_{0}\right| \geq 2 \epsilon\end{cases}
$$

This has an exact, periodic solution to $\Delta w^{\prime}=\rho^{\prime}$

$$
w^{\prime}= \begin{cases}\frac{\left|x-x_{0}\right|^{2}}{6 \epsilon^{3}} & \left|x-x_{0}\right| \leq \epsilon \\ -\frac{\left|x-x_{0}\right|^{2}}{42 \epsilon^{3}}-\frac{8\left|x-x_{0}\right|^{-1}}{21}+\frac{12}{21 \epsilon} & \epsilon<\left|x-x_{0}\right|<2 \epsilon \\ \frac{1}{3 \epsilon} & 2 \epsilon \leq\left|x-x_{0}\right| .\end{cases}
$$

Since $w^{\prime}=O\left(\epsilon^{-1}\right)$, it follows that nonlocal term in (62) can be estimated

$$
\int\left|\nabla w^{\prime}\right|^{2} d x=-\int w^{\prime} \Delta w^{\prime}=O\left(\epsilon^{-1}\right)
$$

Therefore the entire second variation can be written

$$
S\left(\rho^{\prime}\right)=C_{1} \epsilon^{-3}\left[\eta\left(\bar{R}\left(x_{0}\right)\right)+O(\epsilon)\right]+C_{2} \epsilon^{-1}+O(1), \quad \epsilon \rightarrow 0
$$

for positive constants $C_{1}, C_{2}$. For $\epsilon$ small enough, the term involving $\eta$ dominates and $S\left(\rho^{\prime}\right)<0$. 


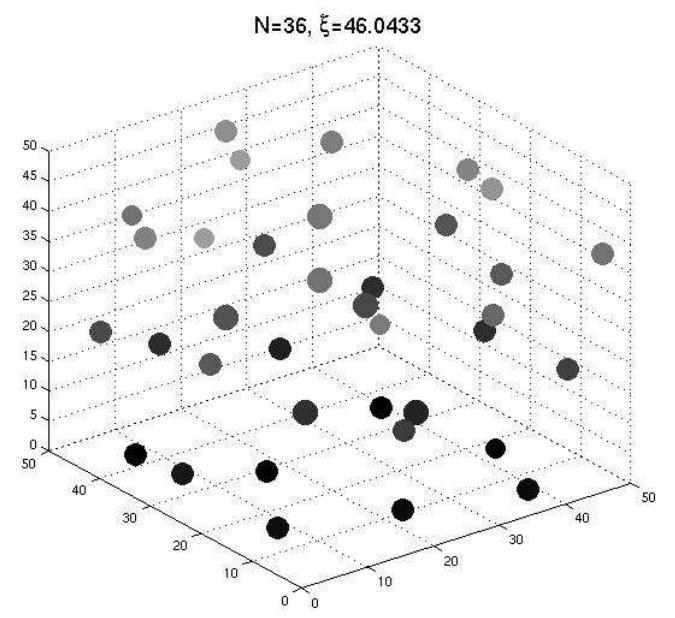

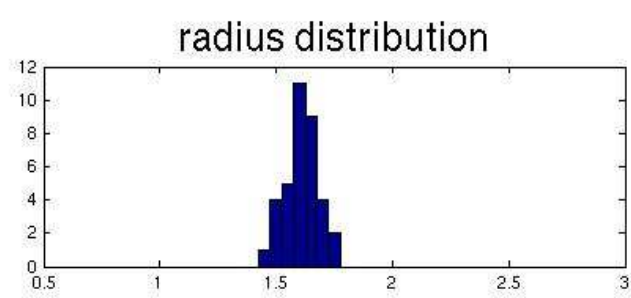

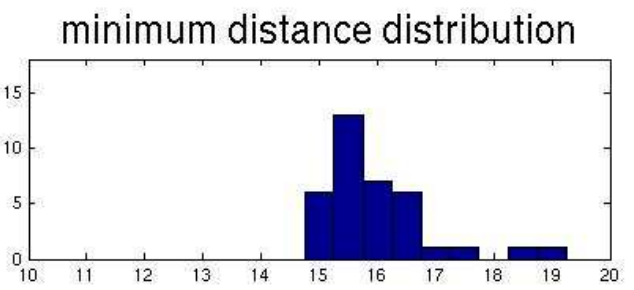

Figure 7: Equilibrium configuration and statistics for large screening length, showing a small variation in sizes (the grey shading indicates $z$-coordinate). Particle sizes are exaggerated for clarity.

4.5. Numerical illustrations. To exhibit the interplay between particle configuration and distribution of radii, the screened approximate system (40-42) was simulated. In the first case, there were $N=36$ particles in a periodic domain of dimensions $[0,50]^{3}$. The locations of the particles were chosen at random, with the constraint that the minimum inter-particle distance is not less than several times the size of the particles. The radii were chosen from a uniform random distribution in the interval $R \in\left[R_{*}, 2 R_{*}\right]$. The average radius and average minimum distance between particles was used to define the screening length. For this case, the result was $\xi \approx 46$, which is same order as the domain size.

The equilibrium configuration is shown in Figure 7 , together with the statistics for radius and minimum spacing distributions. The notable feature is that the distribution of radii is sharply peaked about $R \approx 1.6$.

To yield small screening length, $N=297$ particles were also simulated, giving $\xi \approx 6.3$ which is much smaller than the domain size. The equilibrium configuration and statistics are shown in Figure 8. In this case, the radii distribution exhibits are larger variance in contrast to the large screening length case. This is a reflection of the fact that particles here only interact locally on the scale $\xi$, so there is greater opportunity to have spatial variations in particles sizes.

\section{Particle migration}

Near the $i$-th spherical particle, the boundary layer may be further approximated as

$$
v(x)=\frac{A_{i}}{4 \pi\left|x-x_{i}\right|}+v_{1}(x)+o(1), \quad R \rightarrow 0 .
$$



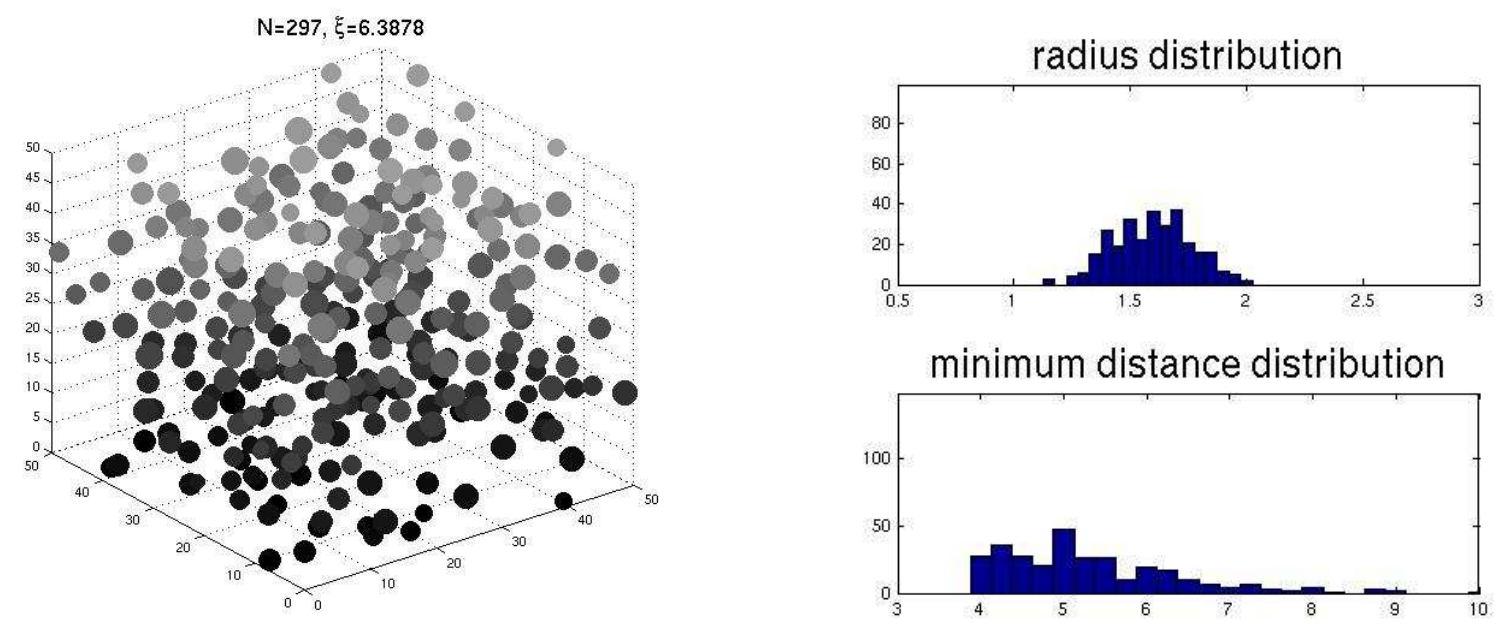

Figure 8: Equilibrium configuration and statistics for small screening length, showing a larger variation in sizes due to less interaction between widely spaced particles.

The term $v_{1}$ is a $O(1)$ subdominant correction, which we will show is responsible for migration of particles.

A systematic matching of $v$ to the monopole solution (12) means that $v_{1}$ will solve the following problem:

$$
\Delta v_{1}=0, \quad v_{1}=0 \text { when }\left|x-x_{i}\right|=R_{i}, \quad \lim _{|x| \rightarrow \infty} \nabla v_{1} \cdot \mathbf{n}=\psi \cdot \mathbf{n},
$$

for any direction $\mathbf{n}$ where

$$
\psi=\sum_{j \neq i} A_{j} \nabla G_{p}\left(x_{i}-x_{j}\right)
$$

Looking for solutions of the form $v_{1}=\Phi\left(\left|x-x_{i}\right|\right) \cos (\varphi)$, where $\varphi$ is the (polar) angle between $x-x_{i}$ and $\psi$, one obtains

$$
v_{1}=|\psi|\left(\left|x-x_{i}\right|-R_{i}^{3}\left|x-x_{i}\right|^{-2}\right) \cos (\varphi) .
$$

Since $v_{1}=0$ inside the sphere, the corresponding free boundary velocity is

$$
\frac{\partial v_{1}}{\partial n}=3|\psi| \cos (\varphi)
$$

which corresponds to a rigid translation of a sphere. It follows that the particle velocity can be written

$$
\frac{d x_{i}}{d t}=3 \sum_{j \neq i} A_{j} \nabla G_{p}\left(x_{i}-x_{j}\right) .
$$


Observe that the radial dynamics (22) have an associated timescale

$$
\tau_{R} \sim \frac{R}{d R / d t} \sim R^{3}
$$

If the screening length is comparable to the system size, the summation in (71) can be estimated as an integral like equation (15) so that the migration dynamics have the timescale

$$
\tau_{X} \sim \frac{d}{d x / d t} \sim R^{4 / 3}
$$

A similar argument is given below for small screening length. Therefore one expects migration of particles to be much slower than the dynamics which causes equilibration of radii. In particular, this justifies the study of particle distributions in which their positions are held steady. In systems larger than the screening length, this means that the particle radius distribution is well approximated by a steady solution of (58) at fixed time.

5.1. Variational characterization. We can amend the discussion of section 3.3 by including subdominant terms in the approximation of the nonlocal energy term. These extra terms represent the interaction energy of particles which gives rise to the migration dynamics.

To account for inter-particle interactions, the field $w$ is better approximated as

$$
w= \begin{cases}\frac{\left|x-x_{i}\right|^{2}}{6}-\frac{R^{2}}{2} & \text { on each } B_{R_{i}}\left(x_{i}\right) \\ -\sum_{i=1}^{N} V_{i} G_{p}\left(x-x_{i}\right) & \text { in } \Omega^{-},\end{cases}
$$

where $V_{i}=4 \pi / 3 R_{i}^{3}$ makes the normal derivatives on $\partial \Omega$ continuous. The nonlocal part of the total energy can be written

$$
\begin{aligned}
\int_{\Omega}|\nabla w|^{2} d x & =\sum_{i=1}^{N}\left(\int_{B_{R_{i}}\left(x_{i}\right)}|\nabla w|^{2} d x+V_{i}^{2} \int_{\Omega / B_{R_{i}}\left(x_{i}\right)}\left|\nabla G_{p}\left(x-x_{i}\right)\right|^{2} d x\right) \\
& +\sum_{i=1}^{N} \sum_{j=1, j \neq i}^{N} V_{i} V_{j} \int_{\Omega^{-}} \nabla G_{p}\left(x-x_{i}\right) \cdot \nabla G_{p}\left(x-x_{j}\right) d x .
\end{aligned}
$$

The first two terms represent the nonlocal parts of each particle's self energy (27). The last term is computed by writing

$$
\begin{aligned}
& \int_{\Omega^{-}} \nabla G_{p}\left(x-x_{i}\right) \cdot \nabla G_{p}\left(x-x_{j}\right) d x=\int_{\Omega^{-}} G_{p}\left(x-x_{i}\right) d x \\
& +\int_{\partial \Omega} G_{p}\left(x-x_{i}\right) \nabla G_{p}\left(x-x_{j}\right) \cdot n d x,
\end{aligned}
$$

where $n$ is the inward normal with respect to $\Omega^{+}$. The first term is independent of $x_{i}$, and in view of (11),

$$
\int_{\Omega^{-}} G_{p}\left(x-x_{i}\right) d x=E_{0}+O\left(R^{2}\right), \quad R \rightarrow 0,
$$


where $E_{0}$ is a constant which ultimately depends on the choice of gauge for $G_{p}$. Near the $j$-th particle $G_{p}\left(x-x_{i}\right) \approx G_{p}\left(x_{j}-x_{i}\right)$ so that

$$
\begin{aligned}
\int_{\Omega^{-}} \nabla G_{p}\left(x-x_{i}\right) \cdot \nabla G_{p}\left(x-x_{j}\right) d x & =E_{0}+G_{p}\left(x_{j}-x_{i}\right) \int_{\partial B_{R_{i}}\left(x_{i}\right)} \nabla G_{p}\left(x-x_{j}\right) \cdot n+O\left(R^{2}\right) \\
& =E_{0}+G_{p}\left(x_{j}-x_{i}\right)+O\left(R^{2}\right) .
\end{aligned}
$$

It follows that the total energy in the limit of small $R$ is approximated as $E=E_{s}+E_{I}$ where the interaction energy is

$$
E_{I}=\frac{\gamma}{2} \sum_{i=1}^{N} \sum_{j=1, j \neq i}^{N} V_{i} V_{j}\left[E_{0}+G_{p}\left(x_{j}-x_{i}\right)\right]
$$

Notice that in the structured regime $\gamma \sim R^{-3}, E_{s} \sim R^{2}$ and $E_{I} \sim R^{3}$. Therefore (since kinetics for migration and mass exchange both scale as $R^{3}$ ), the motion associated with $E_{I}$ should be subdominant to the radial dynamics associated with $E_{s}$. This is another justification of the separation of timescales discussed earlier.

On the very slow timescale associated with migration, the radii are all the same and constant in time, so according to (23) $A_{i}=-\gamma V_{i}=$ constant. The dissipation of $E_{I}$ is computed using (71) as

$$
\begin{aligned}
\frac{d E_{I}}{d t} & =\frac{\gamma}{2} \sum_{i=1}^{N} \sum_{j=1, j \neq i}^{N} V_{i} V_{j}\left(\nabla G_{p}\left(x_{j}-x_{i}\right) \cdot \frac{d x_{j}}{d t}-\nabla G_{p}\left(x_{j}-x_{i}\right) \cdot \frac{d x_{i}}{d t}\right) \\
& =-\frac{1}{3} \sum_{i=1}^{N} V_{i} \frac{d x_{i}}{d t} \sum_{j=1, j \neq i}^{N}\left(-3 \gamma V_{j} \nabla G_{p}\left(x_{i}-x_{j}\right)\right) \\
& =-\frac{1}{3} \sum_{i=1}^{N} V_{i}\left(\frac{d x_{i}}{d t}\right)^{2} .
\end{aligned}
$$

This quantity can be interpreted as a Riemannian metric associated with a gradient flow. The chemical potential $\mu$ needed to create a migration velocity $d x_{i} / d t$ of an isolated particle satisfies

$$
\Delta \mu=0, \quad\left[\frac{\partial \mu}{\partial \mathbf{n}}\right]_{+}^{-}=\frac{d x_{i}}{d t} \cdot n \text { when }\left|x-x_{i}\right|=R_{i}, \quad \nabla \mu \rightarrow 0,|x| \rightarrow \infty .
$$

The solution is

$$
\mu= \begin{cases}-\frac{1}{3}\left(x-x_{i}\right) \cdot \frac{d x_{i}}{d t} & \left|x-x_{i}\right|<R_{i} \\ \frac{R_{i}^{3}}{3} \frac{\left(x-x_{i}\right) \cdot\left(d x_{i} / d t\right)}{\left|x-x_{i}\right|^{3}} & \left|x-x_{i}\right|>R_{i}\end{cases}
$$

so that the dissipation of energy $\int|\nabla \mu|^{2} d x$ of a collection of approximately isolated particles gives is precisely (78). 
5.2. Connection with the Next Order Gamma-convergence of the Energy. Following the discussion of Section 3.4, we can also use Gamma-convergence [5] to support the energetic findings of the previous section. In Section 3.4 we saw that the first order asymptotics of the rescaled energy, $F_{\eta}$ is given by a local functional $F_{0}$, defined on weighted sums of Dirac masses, $\sum_{i} m^{i} \delta_{x_{i}}$. To find the next order, one considers the asymptotics as $\eta \rightarrow 0$ (i.e. the Gamma-limit) of

$$
F_{\eta}-\min \left(\eta^{-1 / 3} F_{0}\right)
$$

In doing so, one finds an interaction energy of the form:

$$
C_{0} \sum_{i}\left(\alpha^{i}\right)^{2}+\sum_{(i, j): i \neq j} \alpha^{i} \alpha^{j} G_{p}\left(x^{i}-x^{j}\right),
$$

where $C_{0}$ is a constant associated with $G_{p}$ (see [5] for details). As we argued in Section 3.4 , we may relate $\alpha^{i}, \eta, \rho$ to the radii and volume of the small particles. In doing so and incorporating the scaling $\gamma \sim 1 / \eta$, we see that the dominant second term of (81) and that of $E_{I}$ in $(77)$ are identical.

5.3. Homogenization and kinetic equations. For a large particle numbers, the migration given by (71) approximates an integral which can be treated by homogenization as in Section 4. In particular, using (47) we have

$$
\sum_{j \neq i} A_{j} \nabla G_{p}\left(x_{i}-x_{j}\right) \approx 4 \pi \int_{\Omega}[2 \phi(y)-\mu(y) \bar{v}(y)] \nabla G_{p}\left(x_{i}-y\right) d y .
$$

Taking the gradient of the integral form of the homogenized equation (53), it follows that the dynamics given by (71) in the homogenized limit is

$$
\frac{d x_{i}}{d t}=3 \nabla \bar{v}\left(x_{i}\right)
$$

Migration of particles can be incorporated into the kinetic equation (56) by noting the probability flux due to migration is just $\nu \nabla \bar{v}$, so that the joint density $\nu$ satisfies

$$
\nu_{t}+\partial_{R}\left(R^{-2}\left[R \bar{v}-2-\gamma R^{3} / 3\right] \nu\right)+\nabla_{x} \cdot(3 \nu \nabla \bar{v})=0 .
$$

Since the mean field $\bar{v}$ scales like $1 / R$ (by virtue of the boundary condition), and $\bar{v}$ varies over spatial length scales of $\xi$, the velocity given by (83) scales like $R^{-1} \xi^{-1}$. The associated timescale is therefore

$$
\tau_{X} \sim \frac{d}{d x / d t} \sim R^{4 / 3} \xi^{5 / 3}
$$

Not only is migration slower than mass exchange, but screening apparently inhibits motion. On timescales larger than $R^{-3}$, particle radii are determined quasi-statically by solving (58) for a given number density $\phi$. Therefore using the characterization (57) of quasi-steady radii distributions, the evolution of $\phi$ can be written as the closed system

$$
\phi_{t}+\nabla_{x} \cdot(3 \nu \nabla \bar{v})=0
$$

where $\bar{v}$ solves (58). 

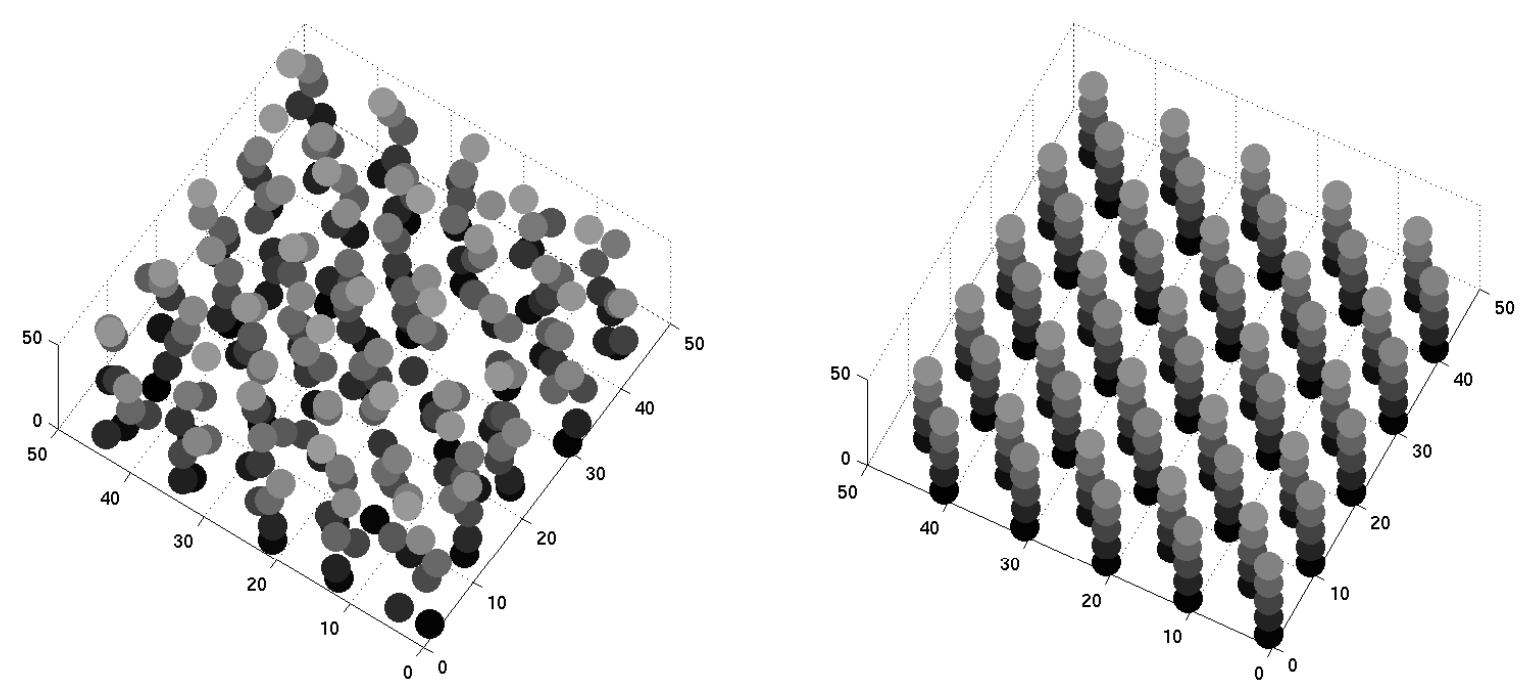

Figure 9: Initial and final configuration of 250 particles. Repulsive dynamics given by the approximation (71) leads to the formation of a regular BCC lattice.

5.4. Computational results. We now illustrate the migration and self-organization of particles using the finite dimensional approximation (71). Figure 9 shows the initial and final configuration of 250 particles on a $[0,50]^{3}$ domain. The radii were all initialized to the critical value $R=R_{*}$. The initial configuration was random, but particles closer than three radii to their neighbors were deleted.

The dynamics described by (71) is repulsive, and the final state is an exact (or nearly exact) body-centered cubic (BCC) lattice, which is expected for strongly segregated dilute copolymer mixtures [1]. The absence of defects in the BCC pattern is partly due to the choice of particle number, which is exactly enough for a $5 \times 5 \times 5$ tiling with the BCC configuration. We have conducted other experiments with an uneven number of particles, and lattice dislocations appear. The nature of these defects is left to future study, however.

\section{REMARKS ON THE TWO-DIMENSIONAL THIN FILM GEOMETRY}

A similar scheme to reduce the dynamics to a set of ODEs can be accomplished in two dimensions, although the results are more subtle because of the presence of a logarithmic boundary layer [4]. Nevertheless, even rigorous results have been obtained for the limit of small volume fraction for the classical Ostwald system [18]. The hypothesis leading to those results only depend on particles being well-separated and having radii of a similar size, which is what we expect here as well.

The Green's function solving (10) on the two dimensional domain $\Omega=[0,1]^{2}$ with periodic boundary conditions has the form

$$
G_{p}(x)=-\frac{1}{2 \pi} \ln |x|+O(1), \quad|x| \rightarrow 0 .
$$


It then suffices to construct an approximation for $x \in \Omega_{-}$

$$
v=A_{0}-\sum_{i=1}^{N} \frac{A_{i}}{2 \pi} \ln \left|\frac{x-x_{i}}{d}\right|+O(1), \quad \sum_{i=1}^{N} A_{i}=-\gamma \rho,
$$

where $d$ is a cutoff length scale, chosen to be the typical distance between particles. The reasoning for this is a follows: at a distance $\sim d$ from particles, the field $v$ should behave like the mean field, represented by $A_{0}$. Although the choice of $d$ is somewhat arbitrary, we show below that the leading order in the approximation is ultimately unaffected.

Applying the boundary condition to the $i$-th particle, one has

$$
\frac{1}{R_{i}}=A_{0}+\frac{A_{i}}{2 \pi} \ln \left(d / R_{i}\right)+O(1), \quad R_{i} / d \rightarrow 0 .
$$

Because of the choice of $d$ as the cutoff, the other terms in the sum in (87) are (logarithmically) subdominant to $\ln \left(d / R_{i}\right)$. Notice that the logarithmic term

$$
L_{\rho}=\ln \left(d / R_{i}\right)=\ln \left(\rho^{-1 / 2}\right)+O(1), \quad \rho \rightarrow 0,
$$

provided the radii satisfy $R_{i} /\left(d \rho^{1 / 2}\right)=O(1)$ as $\rho \rightarrow 0$. This is why it suffices to regard $d$ as an arbitrary measure of particle distance. Accordingly, the approximation $L_{\rho} \equiv \ln \left(\rho^{-1 / 2}\right)$ is used from here on.

For $x \in \Omega_{+}$, the solution has the form

$$
v=C+\frac{\gamma\left|x-x_{i}\right|^{2}}{4}
$$

so that using (88) the radial dynamics are

$$
\frac{d R_{i}}{d t}=-\frac{A_{i}}{2 \pi R_{i}}-\frac{\gamma R_{i}}{2}=\frac{A_{0} R_{i}-1-\gamma L_{\rho} R_{I}^{3} / 2}{R_{i}^{2} L_{\rho}} .
$$

The second requirement in (87) implies, using (88) that

$$
A_{0}=\frac{\sum_{i=1}^{N} 1 / R_{i}+\left(\gamma L_{\rho} / 2\right) \sum_{i=1}^{N} R_{i}^{2}}{N} .
$$

The behavior of the system (90)-(91) is similar to the three dimensional case. In particular, for small radii initial conditions, the critical radius where the growth interval vanishes is computed to be

$$
R_{*}=\left(\frac{1}{\gamma L_{\rho}}\right)^{1 / 3}
$$

The interesting difference from three dimensions is that the equilibrium radius depends on the volume fraction. 


\section{DisCussion}

This work has demonstrated that evolution of dilute copolymer mixtures can be analyzed by an approximation scheme similar to those of ordinary dilute mixtures. The principal differences arise in the slowing of the coarsening process and the ultimate migration toward spatially ordered patterns. We note that the problem studied here has various other physical analogs, including ferrofluids [26] and Langmuir monolayers [29]. These problems have the common ingredients of short-ranged attraction, which creates phases, and long-ranged repulsion, that results in phase patterning. It is expected that our results would apply to these cases as well.

The approach taken in this article has been primarily formal, and it is natural to seek out rigorous results to support our findings. Starting with an ansatz of small spherical particles, we have essentially focused on two dynamic regimes, the first related to coarsening and the second related to migration and self-organization. As we have already mentioned, there is a direct connection here with the rigorous asymptotics via Gamma-convergence of the energy in the limit of small volume fraction. The dynamics of the first regime are driven via a self energy which is simply the leading order term of the asymptotics (the first order Gamma-limit), while the dynamics of the second regime are driven by a Coulomb-like interaction energy which is the next order term in the asymptotics. It would be interesting to further justify our dynamic equations (gradient flows) via the recently established connection between Gamma-convergence and gradient flows (cf. [28]).

There is also rigorous work on stable spherical patterns displaying this dichotomy of energetic effects. In [25], Ren and Wei prove the existence of sphere-like solutions to the Euler-Lagrange equation of (1) and further investigate their stability. Working in a regime of small volume fraction, they also show that the centers of sphere-like solutions are close to global minimizers of an effective energy defined over point masses which includes both a local energy defined over each mass and a Green's function interaction term which sets the location of the approximate spheres.

In terms of the first regime of coarsening, one would like to have some rigorous statement concerning the threshold for particles of critical radius size $R_{*}$ whereby particle systems of radii $R$ where $R>R_{*}$ constitute local minimizers (stationary states) with respect to some underlying topology. One approach could be to interpret the kinetic equation as gradient flow with respect to some topology, and show that delta mass distributions $\nu=\delta_{R}, R>R_{a}$ st are local minimizers with respect to the underlying topology. This is the approach taken in [13] via the Wasserstein metric. Another approach could be via the method of Kohn and Otto [15] on rigorous bounds on scaling laws for the particle radius, where one might be able to show cross over of different bounds (or a collapse altogether) as the length scale approached the critical value. 


\section{Appendix A: Stability of a Single Particle}

An isolated particle can be regarded as a steady solution to

$$
\begin{aligned}
\Delta v & =\gamma \begin{cases}1 & \text { in } \Omega^{+} \\
0 & \text { in } \Omega^{-}\end{cases} \\
v & =\kappa \text { on } \partial \Omega^{+} \\
V_{n} & =[\partial v / \partial n]_{+}^{-} \\
v(x) & \sim \frac{\gamma}{4 \pi|x|^{2}}\left|\Omega^{+}\right|, \quad|x| \rightarrow \infty
\end{aligned}
$$

(The last condition arises from mass conservation). The energy corresponding to this version of our problem is

$$
F=\int_{\partial \Omega^{+}} d A+\frac{\gamma}{2} \int_{\Omega}|\nabla w|^{2} d x
$$

where $w$ is a solution to

$$
\Delta w= \begin{cases}1 & \text { in } \Omega^{+} \\ 0 & \text { in } \Omega^{-}\end{cases}
$$

The radially symmetric particle solution to (93-95) of radius $R$ has

$$
w= \begin{cases}\frac{|x|^{2}}{6} & |x|<R \\ \frac{R^{3}}{3|x|} & |x|>R\end{cases}
$$

Stability is computed by the second variation of the energy; a calculation similar to Choksi \& Sternberg [8] gives the following criteria for stability:

$$
\begin{aligned}
J(\zeta) \equiv & \int_{\partial B_{R}}\left|\nabla_{s} \zeta\right|^{2}-2 R^{-2} \zeta^{2} d s \\
& +\gamma\left(\int_{\partial B_{R}} \int_{\partial B_{R}} G(x-y) \zeta(x) \zeta(y) d s(x) d s(y)-\frac{R}{3} \int_{\partial B_{R}} \zeta^{2} d s\right) \geq 0
\end{aligned}
$$

for smooth test functions $\zeta$ on the spherical surface with zero average. The notation $\nabla_{s}$ refers to the gradient on the surface $\partial B_{R}$ and $G$ is the free space Green's function.

An expansion in terms of spherical harmonics

$$
\zeta=\sum_{l=1}^{\infty} \sum_{|m| \leq l} C_{l m} Y_{l}^{m}(\theta, \phi)
$$

allows the bilinear form (101) to be diagonalized. (Recall these form a complete orthonormal set on $L^{2}(\partial B)$ with eigenvalues $\left.-\Delta_{s} Y_{l}^{m}=l(l+1) Y_{l}^{m}\right)$. The nonlocal term in (101) is computed by letting $\mu=\zeta(\theta, \phi) \delta(r-R)$ and $w$ be a weak solution (in $R^{3}$ ) to

$$
-\Delta w=\mu \text {. }
$$


so that

$$
\int_{\partial B_{R}} \int_{\partial B_{R}} G(x-y) \zeta(x) \zeta(y) d s(y) d s(x)=\int_{\partial B_{R}} w \zeta d s .
$$

Equation (102) can be solved by eigenfunction expansions, giving

$$
w=\sum_{l=1}^{\infty} \sum_{|m| \leq l} R^{1-\alpha} \frac{C_{l m}}{2 l+1} r^{\alpha} Y_{l}^{m}(\theta, \phi)
$$

where $\alpha=l$ for $r<R$ and $\alpha=-l-1$ for $r>R$. Utilizing orthogonality,

$$
J(\zeta)=\sum_{l=1}^{\infty} \sum_{|m| \leq l} C_{l m}^{2}\left(l(l+1)-2+R^{3} \gamma\left(\frac{1}{2 l+1}-1 / 3\right)\right) .
$$

It follows that stability requires

$$
l(l+1)-2+R^{3} \gamma\left(\frac{1}{2 l+1}-1 / 3\right) \geq 0
$$

for $l=1,2,3, \ldots$ The modes corresponding to $l=1$ give $J=0$ since the energy has translational symmetry. As $R^{3} \gamma$ is increased, the first unstable modes occur when $l=2$; therefore the spherical particle solution is stable provided

$$
R \leq R_{s} \equiv\left(\frac{30}{\gamma}\right)^{1 / 3}=10^{1 / 3} R_{*}
$$

where the critical radius $R_{*}$ is defined in $(25)$.

\section{Appendix B: Ewald summation of periodic Green's functions}

Here we recap a practical way of approximating periodic Green's functions using Ewald summation techniques. The solution $G_{p}$ to (10) can be found using eigenfunction expansions as

$$
G_{p}(x)=\sum_{k \neq 0} \exp (i 2 \pi k \cdot x)(2 \pi|k|)^{-2}=\sum_{k \neq 0} \int_{0}^{\infty} \exp \left(2 \pi i k \cdot x-(2 \pi|k|)^{2} t\right) d t
$$

where $k \in \mathbb{Z}^{3}$. The main idea is to split the integral at $t=\beta$ in such a way that one part is a quickly converging sum in $k$ (for large $t$ ), whereas the other part (for small $t$ ) can be rewritten as a quickly converging lattice sum by using the Poisson-Jacobi formula. The result is

$$
G_{p}(x)=\sum_{k \neq 0}(2 \pi|k|)^{-2} \exp \left(-\beta[2 \pi|k|]^{2}\right) \exp (2 \pi i k \cdot x)+\frac{1}{4 \pi} \sum_{K} \frac{\operatorname{erfc}(|x-K| /(2 \beta))}{|x-K|}
$$

where $K \in \mathbb{Z}^{3}$. The optimal choice is $\beta \approx L^{2} / 10$ where $L$ is the domain dimension size. 
Acknowledgments: After this work was completed, we became aware of similar work by Helmers, Niethammer and Ren. In [13], the authors construct approximations using a gradient flow of the system constrained to particle-like configurations, in contrast to doing the approximation at the level of the interface dynamics.

KG was supported by NSF awards DMS-0405596 and DMS-0807423. RC was partially supported by an NSERC (Canada) Discovery Grant.

\section{REFERENCES}

[1] Bates, F.S. and Fredrickson, G.H.: Block Copolymers - Designer Soft Materials. Physics Today, 52-2, 32-38 (1999).

[2] Braides, A.: Г-Convergence for Beginners, Oxford Lecture Series in Mathematics and Its Applications, 222002.

[3] Cahn, J.W. and Hilliard, J.E.: Free Energy of a Nonuniform System I. Interfacial Free Energy. J. Chem. Phys., 28(2), 258-267 (1958).

[4] Chakraverty, B.K.: Grain Size Distribution in Thin Films. J. Phys. Chem. Solids 28 2401-2412 (1967).

[5] Choksi, R. and Peletier, M.A.: Small Volume Fraction Limit of the Diblock Copolymer Problem I: Sharp Interface Functional". Preprint available at www.math.sfu.ca/ choksi/pub/

[6] Choksi, R., Peletier, M.A., and Williams, J.F.: On the Phase Diagram for Microphase Separation of Diblock Copolymers: an Approach via a Nonlocal Cahn-Hilliard Functional. Submitted (2008). Preprint available at www.math.sfu.ca/ choksi/pub/

[7] Choksi, R. and Ren, X.: On a Derivation of a Density Functional Theory for Microphase Separation of Diblock Copolymers. Journal of Statistical Physics, 113, 151 - 176 (2003).

[8] Choksi, R. and Sternberg, P.: On the First and Second Variations of a Nonlocal Isoperimetric Problem. J. Reine Angew. Math., 611, 75-108 (2007).

[9] de Gennes, P.G.: Dynamics of Fluctuations and Spinodal Decomposition in Polymer Blends, Journal of Chemical Physics 72 (1980).

[10] Fife, P. and Hilhorst, D.: The Nishiura-Ohnishi Free Boundary Problem in the 1D case. SIAM J. Math. Anal., 33-3, 589-606 (2001).

[11] Figari, R., Papanicolaou, G. and Rubinstein, J.: The Point Interaction Approximation for Diffusions in Regions with Small Holes. In Lecture Notes in Biomath. Vol. 70, Springer 75-86 (1987).

[12] Hamley, I.W.: The Physics of Block Copolymers. Oxford Science Publications, 1998.

[13] Helmers, M., Niethammer, B., and Ren, X.: Evolution in Off-critical Diblock Copolymer Melts. preprint (2008).

[14] Henry, M., Hilhorst, D., and Nittier, Y.: Singular Limit of a Second Order Nonlocal Parabolic Equation of Conservative Type Arising in the Micro-phase Separation of Diblock Copolymers. Hokkaido Math. J. 32 (2003), no. 3, 561-622.

[15] Kohn, R.V. and Otto, F.: Upper Bounds on Coarsening Rates. Comm. Math. Phys. 229 (2002), no. $3,375-395$.

[16] Lifshitz, I.M. and Slyozov, V.V.: The Kinetics of Precipitation from Supersaturated Solid Solutions. J. Chem. Phys. Solids 19 35-50 (1961).

[17] Niethammer, B.: Derivation of the LSW-theory of Ostwald Ripening by Homogenization Methods. Arch. Rational Mech. Anal. 147-2 119-178 (1999).

[18] Niethammer, B. and Otto, F.: Domain Coarsening in Thin Films. Comm. Pure Appl. Math. 54-3 361-384 (2001).

[19] Niethammer, B. and Otto, F.: Ostwald Ripening: The Screening Length Revisited. Calc. Var. Partial Differential Equations 13-1 33-68 (2001).

[20] Niethammer, B. and Valazaquez. Screening in Interacting Particle Systems. Arch. Rational Mech. Anal. 180-3 493 - 505 (2006). 
[21] Nishiura, Y. and Ohnishi, I.: Some Mathematical Aspects of the Micro-phase Separation in Diblock Copolymers. Physica D 84 31-39 (1995).

[22] Ohnishi, I., Nishiura, Y., Imai, M., and Matsushita, Y.: Analytical Solutions Describing the Phase Separation Driven by a Free Energy Functional Containing a Long-range Interaction Term. CHAOS 9-2 329 - 341 (1999).

[23] Ohta, T. and Kawasaki, K.: Equilibrium Morphology of Block Copolymer Melts, Macromolecules 19 2621-2632 (1986).

[24] Otto, F., Rump, T., Slepcev, D.: Coarsening Rates for a Droplet Model: Rigorous Upper Bounds. SIAM J. Math. Anal. 38-2 503-529 (2006).

[25] Ren, X. and Wei, J.: Spherical Solutions to a Nonlocal Free Boundary Problem From Diblock Copolymer Morphology. SIAM J. Math. Anal. 39-5 1497-1535 (2008).

[26] Rosensweig, R.E.: Ferrohydrodynamics. Cambridge University Press, 1985.

[27] Sagui, C. and Desai, R.C.: Effects of Long-range Repulsive Interactions on Ostwald Ripening. Phys. Rev. E. 52-3 2822-2840 (1995).

[28] Sandier, E. and Serfaty, S.: $\Gamma$-convergence of Gradient Flows with Applications to Ginzburg-Landau. Comm. Pure Appl. Math. 57-12 1627-1672 (2004).

[29] Seul, M., Morgan, N.Y., and Sire, C.: Domain Coarsening in a Two-dimensional Binary Mixture. Phys. Rev. Lett., 73(17) 2284-2287 (1994).

[30] Voorhees, P.: The Theory of Ostwald Ripening. J. Stat. Phys. 231-252 (1985).

[31] Wagner, C.: Theorie for Alterung von Niederschlagen durch Umlosen. Z. Elektrochemie 65 581-594 (1961).

Karl Glasner, Department of Mathematics, University of Arizona

E-mail address: kglasner@math.arizona.edu

Rustum Choksi, Department of Mathematics, Simon Fraser University

E-mail address: choksi@math.sfu.ca 